Available online on 15.01.2020 at http://jddtonline.info
Open Access to Pharmaceutical and Medical Research
unrestricted non-commercial use, provided the original work is properly cited

Open@ Access

Review Article

\title{
Psychotic Disorders, Definition, Sign and Symptoms, Antipsychotic Drugs, Mechanism of Action, Pharmacokinetics \& Pharmacodynamics with Side Effects \& Adverse Drug Reactions: Updated Systematic Review Article
}

\author{
Rohit Bangwal1*, Shivam Bisht ${ }^{2}$, Saurabh Saklani2, Shobit Garg33, Mohan Dhayani ${ }^{3}$ \\ 1. PharmD (PB) Intern, Department of Pharmacy Practice, School of Pharmaceutical Sciences, Shri Guru Ram Rai University, Dehradun- \\ 248001, Uttarakhand, INDIA. \\ 2. PharmD, Department of Pharmacy Practice, School of Pharmaceutical Sciences, Shri Guru Ram Rai University, Dehradun-248001, \\ Uttarakhand, INDIA. \\ 3. Department of Psychiatric Medicine, Shri Guru Ram Rai Institute of Medical \& Health Sciences, Patel Nagar, Dehradun-248001, \\ Uttarakhand, INDIA.
}

\begin{abstract}
Psychosis is a mental disorder characterized by a disconnection from reality. Psychosis is a group of disorder characterized by thought disorder, abnormal behaviour, defective cognition, delusion and hallucination. Adverse drug reaction is defined as any undesired or unintend ed effects of drugs treatment. According to the World Health Organization (WHO)- "adverse drug reaction (ADRs) has been defined one which is noxious and unintended, and which occurs at doses normally used in man for prophylaxis, diagnosis, or therapy of disease, or modification of physiological function". Adverse drug reactions are the most important causes of the mortality and morbidity. Antipsychotics are the most effective drugs which are used in the psychiatry in the maintenance therapy of mania, psychoses and schizophrenia. The antipsychotics drugs are chemically disparate but have the common property of alleviating the symptoms of organic as well as functional psychosis. But they also have a capacity to cause a wide range of potential adverse drug reactions that can lead to non-compliance that can impair quality of life, may cause the extra pyramidal symptoms which can lead to discontinuation of therapy and in extreme cases it may be fatal. Knowledge of assessment of ADRs due to different antipsychotics is necessary. It helps to choose to safe treatment and reduce the risk of occurrence of ADRs by the clinicians. ADR are often poorly identified and reported in day to day medical practice. As we collect more and more information about ADRs, we need an active surveillance system regarding identification and reporting of ADRs with antipsychotic drugs. On many review articles are read \& ward round participation experiences we find that antipsychotic drugs can have shown a various kind of ADRs. Psychiatrist and clinical pharmacist are need to be made aware of these potentially fatal adverse effects associated with antipsychotic drugs via conduction of patients counseling regarding (drugs, disease, doses \& side effects), quality-based seminars, published medical literature, conferences, learning programs and health care camps.
\end{abstract}

Keywords: Antipsychotic Drugs, WHO, Adverse Drug Reactions, Pharmacovigilance, Psychiatrist.

Article Info: Received 19 Oct 2019; $\quad$ Review Completed 25 Dec 2019; $\quad$ Accepted 29 Dec 2019; $\quad$ Available online 15 Jan 2020

Cite this article as:

Bangwal R, Bisht S, Saklani S, Garg S, Dhayani M, Psychotic Disorders, Definition, Sign and Symptoms, Antipsychotic Drugs, Mechanism of Action, Pharmacokinetics \& Pharmacodynamics with Side Effects \& Adverse Drug Reactions: Updated Systematic Review Article, Journal of Drug Delivery and Therapeutics. 2020; 10(1):163-172 http: //dx.doi.org/10.22270/jddt.v10i1.3865

*Address for Correspondence:

Rohit Bangwal, PharmD (PB) Intern, Department of Pharmacy Practice, School of Pharmaceutical Sciences, Shri Guru Ram Rai University, Dehradun-248001, Uttarakhand (India)

\section{INTRODUCTION}

A psychosis is a mental disorder characterized by a disconnection from reality. A psychosis is a group of disorder characterized by thought disorder, abnormal behaviour, defective cognition, delusion and hallucination. Adverse drug reactions are the most important causes of the mortality and morbidity. The term psychosis refers to a severe form of mental disorder where there is gross disorganization of ISSN: 2250-1177

[163] personality and severe impairment in social functions. These patients fail to realize what is real and what is not. Reasoning, judgment and insight are lost. Hallucinations and delusions may occur schizophrenia, delirium, dementia, mania, and depression are examples of psychosis ${ }^{1}$. 


\section{CLLASIFICATION OF PSYCHOTIC DISORDER:}

\section{Acute and chronic organic brain syndromes} (Cognitive Disorder):

Such as delirium and dementia with psychotic features, some toxic or pathological basis can often be defined. Prominent features are confusions, disorientation, defective memory, disorganized thought and behaviour.

\section{Functional disorder:}

No underling cause can be defined, memory and orientation are mostly retained but emotion, thought, reasoning and behaviour are seriously altered.

i. Schizophrenia (Split Mind): Schizophrenia is characterized by delusion, hallucination and lack of insight. Acute schizophrenia may also present with disturbed behaviour, disturbed thinking, or with insidious social withdrawal and other so called negative symptoms and less obvious delusion and hallucination.

* There is no anatomical and pathological defect in the brain.

* Psychosis is due to functional impairment of the brain.

* The cognition is not impaired.

\section{Clinical Symptoms:}

The symptoms of schizophrenia are generally organized into two main domains: positive and negative (Table 1). Positive symptoms are usually what the public perceives when thinking about schizophrenia: hallucinations, delusions, and disorganization. Hallucinations can affect any of the five senses, but auditory are the most common. Delusions are fixed false beliefs that are persistent despite evidence to the contrary. Delusional subtypes include persecutory, erotomaniac (e.g., delusion of love), delusions of grandeur (having special abilities), or somatic (i.e., delusion of being pregnant in context of negative pregnancy tests). Disorganization can manifest in the patient's speech, thought patterns, or behaviours. Some examples of disorganized speech can include made-up words (neologisms), rhyming words (clang speech), speaking words in a sentence in incorrect order (word salad), and repeating words said to them (echolalia). Examples of disorganized behaviours can include repeating the same activity needlessly (perseveration), repeating someone else's actions (echopraxia), dressing oddly for the setting or season of the year (wearing winter clothes in summer), or other odd behaviours based upon the setting the patient is in. Negative symptoms of schizophrenia include apathy, avolition, blunted affect, and poverty of speech. A decreased ability to experience pleasure (known as anhedonia) and social withdrawal can lead to significant functional impairment, even when positive symptoms are relatively absent in a patient. During acute episodes, positive symptoms may predominate, whereas over the long term it may be negative symptoms that are more troubling and disabling to a patient. A third domain of cognitive symptoms can also present in schizophrenia. Symptoms include concrete thinking, inattention, problems with memory, learning and executive function, as well as conceptual disorganization. When these cognitive symptoms are combined with negative symptoms over the patient's lifespan, significant impairment in function may result. Comorbid depression is also common in schizophrenia and can be difficult to treat, and symptoms can be predictive of relapse. Depressive symptoms may be overlooked in the face of overwhelming positive symptoms or confused with negative symptoms ${ }^{3}$.

Table-1 Positive and Negative Symptoms of Schizophrenia

\begin{tabular}{|c|l|l|}
\hline S no. & Positive Symptoms & Negative Symptoms \\
\hline $\mathbf{1 .}$ & Hallucinations (auditory, visual, or other senses) & Diminished emotional expression (body language) \\
\hline $\mathbf{2 .}$ & Delusions (persecutory, paranoid, grandiose, etc.) & Avolition/psychomotor retardation \\
\hline $\mathbf{3 .}$ & Disorganized thinking/speech & Alogia (decreased speech) \\
\hline $\mathbf{4 .}$ & $\begin{array}{l}\text { Grossly disorganized or abnormal motor behaviour } \\
\text { (including catatonia) }\end{array}$ & Anhedonia (decreased ability to feel pleasure) \\
\hline $\mathbf{5 .}$ & Unusual behaviour & Asociality (lack of socialization) \\
\hline $\mathbf{6 .}$ & Combativeness, agitation, and hostility & Affective flattening \\
\hline $\mathbf{7 .}$ & Ideas of reference & Social Withdrawal \\
\hline
\end{tabular}

ii. Paranoid States: Paranoid States with marked persecutory or other kinds of fixed delusion (false beliefs) and loss of insight in to the abnormality. About one third of patients suffers from this type and are characterized by presence of delusion and hallucinations. However, there is no thought disorder and no incoherent thinking.

iii. Mood (affective) Disorder: Affective disorders are a set of the psychiatric disease, also called as mood disorder. The primary symptoms is change is mood state, may manifest as:

\section{* Bipolar disorder (mania \& depression)}

Mania: elation and irritable mood, reduced sleep, hyperactivity, uncontrollable thought and speech, may be associated with reckless or violent behaviour.

Depression: sadness, loss of interest and pleasure, worthlessness, guilt, physical and mental slowing, melancholia, self-destructive ideation ${ }^{3}$. 
Different types of depression:

\begin{tabular}{|c|l|l|}
\hline S. No. & Type of depression & Characteristic \\
\hline $\mathbf{1 .}$ & Persistent depressive disorder & Patients have depressed mood for at least two years. \\
\hline $\mathbf{2 .}$ & Bipolar disorder & Mood of a person fluctuates from depression to mania. \\
\hline $\mathbf{3 .}$ & Seational affective disorder & $\begin{array}{l}\text { Mood is affected by season and there is a development of } \\
\text { depression during the winter. }\end{array}$ \\
\hline $\mathbf{4 .}$ & Psychotic depression & Patients exhibit signs of psychosis along with depression. \\
\hline $\mathbf{5 .}$ & Perinatal depression & $\begin{array}{l}\text { Woman experience symptoms of major depression during } \\
\text { pregnancy or after delivery. }\end{array}$ \\
\hline
\end{tabular}

Neurosis on the other hand, is a less severe form of mental disorder with minor impairment in social and personality function. Sense of reality, reasoning and insight are retained. Hallucinations and delusions are absent.

Generalized anxiety disorders, conversion and dissociative disorders, obsessive compulsive disorders, and phobic disorders are examples of neurosis.

a. Anxiety: An unpleasant emotional state associated with uneasiness, worry, tension and concern for the future.

b. Phobic states: Fear of the unknown or of some specific objectives, person and situations.

\section{c. Obsessive compulsive disorder (OCD):}

\section{Obsession:}

OCD is an anxiety disorder in which people have unwanted and repeated thoughts, feelings, ideas, sensation (obsession). eg

* Repetitive thought (eg feeling contaminated after touching, an object, doubting weather the store was turned off.)

* Repetitive images (eg recurrent sexually explicit punts)

* Repetitive hand washing

\section{Compulsion:}

Normal repetitive behaviour that feature in everyday life may include bedtime routine, religious, practice and learning a new skill. Behaviour that makes them feels down to do something (compulsions). eg.

* Washing and cleaning including constant hand washing.

* Checking including, checking body parts.

* Repeating including rereading and repeating routine activities like getting up from a chair.

* Mental compulsion, including praying to present harm and mentally reviewing events.

Limited abnormality of thought or behaviour, recurrent intrusive thoughts or ritual-like behaviours which the patients realize are abnormal or stupid but is not able to overcome even a voluntary effort. The obsessions generate considerable anxiety and distress.

d. Reactive depression: Due to physical illness, loss, blow to self-esteem or bereavement, but excessive or disproportionate. e. Post-traumatic stress disorder: varied symptoms following distressing experiences like war, riots, earthquakes etc.

f. Hysterical: Dramatic symptoms resembling serious physical illness, but situational, and always in the presence of other, the patients do not feign but actually undergoes the symptoms, though the basis is only psychic and not physical ${ }^{1}$.

The terms 'organic' vs 'functional' is a dichotomy still alive in psychiatric vocabulary. Antipsychotics are the most effective drugs which are used in the psychiatry in the maintenance therapy of mania, psychoses and schizophrenia ${ }^{2}$. Antipsychotics are the most effective drugs which are used in the psychiatry in the maintenance therapy of mania, psychoses and schizophrenia ${ }^{3}$. The antipsychotics drugs are chemically disparate but have the common property of alleviating the symptoms of organic as well as functional psychosis. But they also have a capacity to cause a wide range of potential adverse drug reactions that can lead to non-compliance that can impair quality of life, may cause the extra pyramidal symptoms (EPS) which can lead to discontinuation of therapy and in extreme cases it may be fatal 4,5 . Antipsychotics drugs have a broad spectrum of therapeutic effect in clinical practice and are generally safer agents. Adverse effects are augmentation of many pharmacological actions of these drugs such as autonomic nervous system, central, cardiovascular, and endocrine system $6,7,8$.

Knowledge of assessment of ADRs due to different antipsychotics is necessary. It helps to choose to safe treatment and reduce the risk of occurrence of ADRs by the Psychiatrist. The second generations of antipsychotic drugs are differed from the first generation as they have a low risk of adverse reactions such as extra pyramidal symptoms (EPS) and also reduce the positive and negative symptoms of schizophrenia. Besides low tendency of exhibiting an Extra pyramidal symptoms (EPS), second generation antipsychotics exhibit their own spectrum of adverse effects including hypotension, seizures, weight gain, increased risk of diabetes mellitus, hyperlipidaemias. These ADRs are not only common but also have a high rate of preventability. The selection of medicines should be targeted for quality improvement patient healthcare system. It can be done by the data use of frequency, severity, probability and preventability. After getting all the information of high-risk drugs through analysis may have significant impact on reducing the $\mathrm{ADRs}^{9,10}$.

Hence, an attempt has been made in this article exhibit an updated analysis of ADRs which are caused by antipsychotics drug and how to manage the ADRs in general outlook and what are the future outcomes in the management of adverse drug reaction ${ }^{11}$. 


\section{CLASSIFICATION OF ANTIPSYCHOTIC DRUGS}

\section{First-Generation Antipsychotics}

- The first-generation antipsychotic drugs (also called conventional, typical, or traditional antipsychotics) are competitive inhibitors at a variety of receptors, but their antipsychotic effects reflect competitive blocking of D2 dopamine receptors. First-generation antipsychotics are more likely to be associated with movement disorders, particularly for drugs that bind tightly to dopaminergic neuroreceptors, such as haloperidol.

\section{- First-Generation Antipsychotics (Typical) (low potency)}

Chlorpromazine (100-800mg/day)

Triflupromazine (50-200mg/day)

Thioridazine (100-400mg/day)

- First-Generation Antipsychotics (Typical) (high potency)

- Fluphenazine (1-10mg/day)

Haloperidol (5-20mg/day)

, Pimozide (2-6mg/day)

, Thiothixene (5-60mg/day)

\section{Second-Generation Antipsychotic Drugs (Atypical)}

- The second-generation antipsychotic drugs (also referred to as "atypical" antipsychotics) have fewer extrapyramidal symptoms (EPS) than the first-generation agents but are associated with a higher risk of metabolic side effects, such as diabetes, hypercholesterolemia, and weight gain. The second-generation drugs appear to owe their unique activity to blockade of both serotonin and dopamine receptors.

Aripiprazole (10-30mg/day),

Clozapine (100-300mg/day),

Molindone (50-70mg/day),

Olanzapine(2.5-20mg/day),

Quetiapine (50-400mg/day),

Risperidone (2-8mg/day),

\section{MECHANISM OF ACTION (FGAs)}

Dopamine Hypothesis: The dopamine hypothesis states that antipsychotics reduce psychotic symptoms by decreasing dopamine activity. It was originally proposed by Arvid Carlsson from Sweden and other basic scientists based on the observation that haloperidol and chlorpromazine raised the levels of dopamine metabolites in dopamine-rich areas of mouse brain. These drugs had inconsistent effects on other neurotransmitters. They interpreted these findings as indicating that these two agents were acting as dopamine antagonists and that self-regulating systems were compensating by increasing dopamine production. Other investigators noted that FGAs inhibit the activation and stereotypical behaviours of rodents that are induced by amphetamine. This effect has been found to be a reasonably reliable predictor of antipsychotics activity in patients. Moreover, the dose-response relationships in animals provide information about the likely clinical dosage range for patients. These behaviours are thought to be mediated by dopamine and have been used as screening devices to identify compounds that are likely to have clinical efficacy as antipsychotics. Other evidence supporting a role of dopamine in psychosis comes from the observation that all drugs-at least to this date-capable of reducing psychosis are also dopamine antagonists, including partial dopamine agonists. Moreover, agents that are associated with increased dopamine activity such as amphetamine, methylphenidate, or cocaine tend to increase dopamine neurotransmission and also tend to worsen patients with schizophrenia who are in clinically unstable conditions $4,7,8$.

According to the dopamine theory of schizophrenia, positive symptoms are the result of an over activity in the mesolimbic dopamine pathway. This is in part based on the observation that drugs that increase dopaminergic availability (L-DOPA, cocaine, amphetamines) can trigger psychotomimetic effects in individuals not affected by schizophrenia first-generation antipsychotics are D2 antagonists. As a result, they reduce dopaminergic neurotransmission in the four dopamine pathways 9 .

Effects of First-Generation Antipsychotics on the Four Dopamine Pathways (Shown Table 2)

1. Nigrostriatal Pathway: Extrapyramidal Symptoms: In which fibres originate from the A9 region of the pars compacta and project rostrally to become widely distributed in the caudate nucleus and the putamen. One of the major functions of dopamine in nigrostriatal pathway is movements. Antagonism of D2 receptors in the nigrostriatal pathway is associated with increased risk of extrapyramidal symptoms 4,8 .

2. Tuberoinfundibular Pathway: Hyperprolactinemia: This originates in the arcuate nucleus of the hypothalamus and projects to the median eminence. Dopamine acts asprolactin-inhibiting factor or it synonyms with prolactin inhibiting factor in tuberoinfundibular pathways. D2 blockade in this path way increases prolactin levels by promoting its release in the pituitary gland causing hyperprolactinemia ${ }^{4,7}$.

3. Mesocortical Pathway: In which the dopaminergic fibbers also arise from the A10 region (the ventral tegmental area) and project to the frontal cortex and sept hippocampal regions. Schizophrenia pathophysiology suggests that a dysfunction of mesocortical pathway is associated with cognitive impairments and disturbances of emotions and affect (negative symptoms). Blockade of the mesocortical pathway by high doses of first-generation antipsychotics can induce secondary negative symptoms and cognitive effects 4,9 .

4. Mesolimbic Pathway: (Antipsychotic Effects) Where the dopaminergic projections originate in the ventral tegmental area, the A10 region, and then spread to the amygdala, pyriform cortex, lateral septal nuclei and the nucleus accumbens. Over activity of mesolimbic dopamine pathway is thought to be involved in the pathophysiology of positive symptoms of schizophrenia. Blockade of D2 receptors in the mesolimbic pathway has been proposed as a possible mechanism of antipsychotic action of first-generation agents 4,10 . 
TABLE 2 Clinical Effects of Receptor Antagonism by Antipsychotics

\begin{tabular}{|c|c|l|}
\hline S. no. & Receptor (Subtype) & \multicolumn{1}{c|}{ Clinical Effect of Antagonism } \\
\hline $\mathbf{1 .}$ & Dopamine (D2) & $\begin{array}{l}\text { Mesolimbic pathway (basal ganglia)-treatment of positive symptoms } \\
\text { Mesocortical pathway (prefrontal cortex)-may worsen negative symptoms } \\
\text { Nigrostriatal pathway (substantia nigra)-extrapyramidal symptoms (EPS) } \\
\text { Tuberoinfundibular pathway (hypothalamus-anterior pituitary)-increased } \\
\text { prolactin release }\end{array}$ \\
\hline $\mathbf{2 .}$ & Serotonin (5-HT2a) & $\begin{array}{l}\text { Treatment of negative symptoms; } \\
\text { increased dopamine release in prefrontal cortex; } \\
\text { may reduce mesolimbic dopamine release }\end{array}$ \\
\hline $\mathbf{3 .}$ & Serotonin $(5-\mathrm{HT} 2 \mathrm{c})$ & Implicated in antipsychotic-associated weight gain \\
\hline $\mathbf{4 .}$ & Muscarinic $(\mu 1)$ & $\begin{array}{l}\text { Anticholinergic side effects (dry mouth, constipation, urinary retention, blurred vision, } \\
\text { hot/dry skin, memory impairment); sedation }\end{array}$ \\
\hline $\mathbf{5 .}$ & Histamine $(\mathrm{H} 1)$ & Increased appetite, sedation \\
\hline $\mathbf{6 .}$ & Alpha $(\alpha 1)$ & Orthostatic hypotension \\
\hline
\end{tabular}

\section{MECHANISM OF ACTION (SGAs)}

Most SGAs differ from older medications pharmacologically in that serotonin 5HT2 receptor binding exceeds their affinity for dopamine D2 receptors, whereas in FGAs this is generally not the case. Largely for that reason, 5HT2 activity has been suggested as one basis for the lower overall risk of extrapyramidal side effects (EPS) with the atypical drugs compared with FGAs. Other aspects of SGA pharmacology that correlate with reduced risk of EPS include "loose" D2 receptor binding with rapid dissociation rates and preferential binding of drugs to receptors in limbic and cortical brain regions rather than striatal areas. None of these hypotheses has been fully confirmed, and the most important message for the clinician is that the pharmacology of these drugs is complex and likely to result in some variability of side effect risk and pharmacokinetics from patient to patient 6 .

\section{PHARMACOKINETICS (ADME) OF ANTIPSYCHOTIC DRUGS}

\section{Absorption}

Mostly, all the antipsychotics drugs are readily but incompletely absorbed. Many drugs undergo first pass metabolism. The oral administration of Chlorpromazine \& Thioridazine have systemic availability of $25-35 \%$, whereas Haloperidol systemic availability of about $65 \%$. In general, the FGAs are well absorbed when they are administered orally or parentally. As with most drugs, oral administration leads to less predictable absorption than parenteral administration. Liquid concentrates are absorbed slightly more rapidly than pills. Plasma concentrations of the drugs usually reach peak levels 1 to 4 hours after ingestion and 30 to 60 minutes after intramuscular (IM) administration. In general, IM preparations reach their peak concentrations sooner than oral drugs and as a result have an earlier onset of action. For example, IM administration of most antipsychotics results in peak plasma levels in about 30 minutes with clinical effects emerging within 15 to 30 minutes ${ }^{3,4}$.

\section{Half-Lives and Steady State}

Antipsychotics usually reach steady state in about three to five half-lives. Thus, steady state levels for chlorpromazine, haloperidol, and most other FGAs are reached in about 3 to 5 days since their half-lives are about 24 hours 4 .

\section{Distribution}

Antipsychotic drugs are highly lipid soluble \& protein bound i.e., 92 to $99 \%$. They also have a large volume of distribution (i.e., $>7 \mathrm{~L} / \mathrm{Kg}$ ). They have long duration of action than would be estimated from their plasma half-lives. Symptoms of psychoses will be reoccurrence in 6 months after the discontinuation of treatment ${ }^{4}$.

\section{Bioavailability}

The bioavailability (that is, the amount of drug reaching the systemic circulation) is substantially greater-as much as tenfold when FGAs are administered parenterally. Most FGAs are highly protein bound. For example, more than 90 percent of drugs such as fluphenazine and haloperidol is bound to plasma protein. The remaining unbound portion is the drug that is available for passing through the blood-brain barrier. In theory, conditions that alter the amount of plasma protein such as malnutrition will also alter the amount of bioavailable antipsychotic drug. This is potentially important for drugs that are protein bound for 98 percent or more 4 .

\section{Metabolism and Elimination}

Antipsychotics drugs are metabolized by the oxidation, demethylation, catalysed by liver microsomal CYP-P450 enzymes, CYP2D6, CYP1A2, CYP3A4. The elimination $t 1 / 2$ is variable, but mostly is in the range of $18-30 \mathrm{hrs}$. The metabolites are excreted in urine \& bile weeks after the discontinuing of treatment. The broad spectrum of pharmacokinetics of neuroleptics is similar. Most FGAs are metabolized by the cytochrome P450 2D6 and P450 3A subfamilies in the liver. Since these same isoenzymes also metabolize a number of drugs that are commonly combined with antipsychotics, a number of important drug-drug interactions are possible the systemic clearance of FGAs is high as the result of a high hepatic extraction ratio. As a result, only negligible amounts of the unchanged drug are excreted by the kidneys. Phenothiazines, thioxanthenes, and their metabolites are excreted in the urine and the faeces ${ }^{14}$. (Show Table 3) 
Pharmacokinetic Of Selected First-Generation Antipsychotics

Table 3. Pharmacokinetics of Selected antipsychotics.

\begin{tabular}{|c|c|c|c|c|}
\hline Drug & $\begin{array}{c}\text { Bioavailability } \\
\mathbf{( \% )}\end{array}$ & $\begin{array}{c}\text { Protein binding } \\
\mathbf{( \% )}\end{array}$ & $\mathbf{t}_{\mathbf{1} / \mathbf{2}} \mathbf{( h r s )}$ & Cp 12 hrs Post dose (ng/ml) \\
\hline Chlorpromazine & $10-33$ & $90-95$ & $8-35$ & $100-300$ \\
\hline Thioridazine & $25-33$ & 99 & $9-30$ & $200-800$ \\
\hline Perphenazine & 25 & $90-95$ & $8-21$ & $0.5-5.0$ \\
\hline Fluphenazine & $20-50$ & $90-95$ & $14-24$ & $0.2-3.0$ \\
\hline Haloperidol & $40-70$ & 92 & 36 & $2-18$ \\
\hline Thiothixene & 50 & $90-95$ & 34 & $1-5$ \\
\hline
\end{tabular}

Cp-Concentration in plasma after 12 hours.

\section{PHARMACODYNAMICS OF ANTIPSYCHOTIC DRUGS}

Mechanism of action of all the antipsychotics in the brain is D2 receptors antagonism that has given rise to the hypothesis that schizophrenia and other positive and negative symptoms. Positive symptoms may occur by the dysregulation of dopaminergic circuits with excess dopaminergic activity in mesolimbic pathway and negative symptoms due to the dopaminergic signalling in the mesocortical pathways. The affirmation for the dopamine hypothesis comes from not only the potency of D2 receptor antagonism, but also the effect of D2 agonists such as amphetamine in precipitating psychosis and the effect of dopamine depleting drugs such as reserpine in reducing psychotic symptoms. ${ }^{15}$

For Schizophrenia, antipsychotic drugs are the cornerstone of the pharmacological treatment such as chlorpromazine which is introduced in 1952 as a first-generation antipsychotic drug. The early antipsychotic drugs are also come in the market as Chlorpromazine, Haloperidol, Fluphenazine are referred to as first generation antipsychotics. These drugs are affecting in relieving positive symptoms but also exhibit a extra pyramidal symptoms, tardive dyskinesia. Because of limitation of these agents may lead to introduced of newer antipsychotics drugs in 1990 such as resperidone, olanzepine, quetiapine, etc. Newer antipsychotics are now termed as second-generation antipsychotic drugs which have a low risk of causing a extra pyramidal symptoms, tardive dyskinesia and also have frequency of producing some adverse drug reactions such as weight gain, metabolic changes and associated cardiovascular consequences. 16,17

The first-generation antipsychotics are effective in the treatment of Schizophrenia but also have tendency to exhibit extra pyramidal symptoms and lead to tardive dyskinesia. However, clozapine withdrawal from the market by manufacturers because it have ability to produce agranulocytosis but later it is reintroduced in the market with strict regulations as white blood cell count follow up and other investigations ${ }^{18,19}$ All the antipsychotics are effective by binding on D2/D3 receptors in the ventral straitum. ${ }^{20}$ Both first and second generation antipsychotics is associated with a clear, dose-dependent risk of seizure provocation. The risk of seizure provocation is high with first generation antipsychotic drugs as compared to second generation antipsychotic drugs. The risk of seizure seems higher for clozapine and lowest for Resperidone.21 The majority of adverse effects of antipsychotic drugs are extensions of their pharmacological action, also these are some adverse effects. ${ }^{22}$ In general, antipsychotics drug have better mainstay in the treatment of psychoses and other mental problems but also have tendency to cause adverse effects $^{23}$.

\section{SIDE EFFECTS \& ADRs of ANTIPSYCHOTICS DRUGS}

Adverse drug reaction (ADRs) is defined as any undesired or unintended effects of drugs treatment. According to the World Health Organization (WHO)- "adverse drug reaction (ADRs) has been defined one which is noxious and unintended, and which occurs at doses normally used in man for prophylaxis, diagnosis, or therapy of disease, or modification of physiological function" 1 .

Adverse drug events (ADEs) is an injury resulting from the use of the drugs. Under this definition, the term ADE includes harm caused by the drugs (ADRs \& overdoses) and harm form the use of the drugs (including dose reduction and discontinuous of drug therapy). ADE may results form medication errors but most do not ${ }^{2}$.

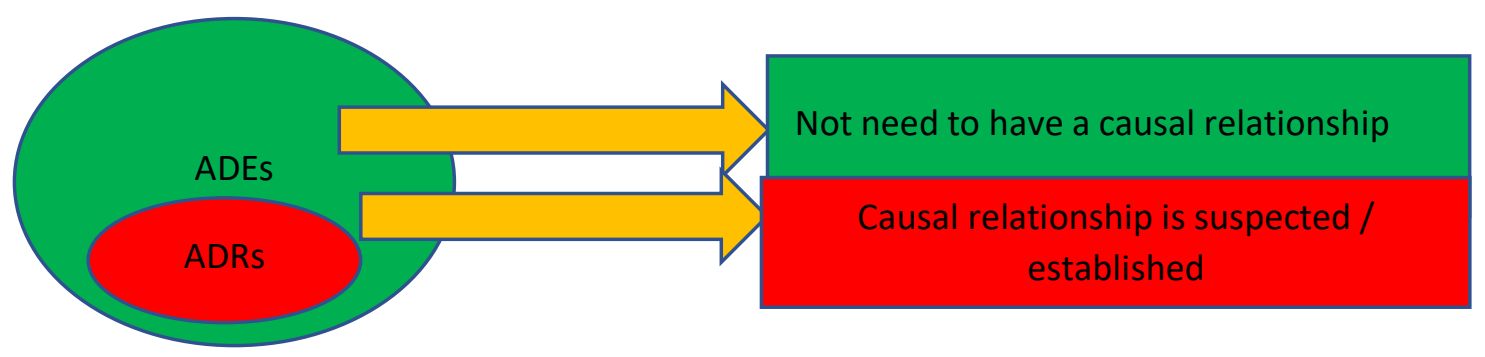

Figure 1- Relationship between ADRs \& ADEs

Treatment with antipsychotic drugs, whether typical or atypical agents, is associated with various adverse effects, including acute EPS (dystonia, parkinsonism and akathisia), chronic EPS (tardive dyskinesia), neuroleptic malignant syndrome, weight gain, glucose intolerance (diabetes mellitus [DM]), metabolic syndrome and elevation of plasma prolactin levels. Several of these side effects are known to be related to distinct pharmacological profiles of the antipsychotic agents. For example, parkinsonism, tardive dyskinesia, neuroleptic malignant syndrome sand 
hyperprolactinemia are considered to be a result of acute or chronic blockade of DA receptors, while histamine- $\mathrm{H}_{1}$ and 5$\mathrm{HT}_{2}$ creceptors may contribute to antipsychotic-induced weight gain.1,3 These are the adverse drug reactions which is associated by antipsychotic drugs are as follows-

\section{A. Central Nervous System \\ 1. Extrapyramidal System \\ a. Dystonia}

- Dystonia are prolonged tonic muscle contractions, (occurring usually within 24-96 hours of dosage initiation or dosage increase); they may be life threatening (eg, pharyngeal-laryngeal dystonia). Other dystonia are trismus, gloss spasm, tongue protrusion, blepharospasm, oculogyric crisis, torticollis, and retro Collis. They occur primarily with FGAs. Risk factors include younger patients (especially male) and use of high-potency agents and high dose.

- Treatment includes IM or IV anticholinergics or benzodiazepines. Benztropine mesylate, $2 \mathrm{mg}$, or diphenhydramine, $50 \mathrm{mg}$, may be given IM or IV, or diazepam, 5 to $10 \mathrm{mg}$ slow IV push, or lorazepam, 1 to $2 \mathrm{mg}$ IM, may be given. Relief usually occurs within 15 to 20 minutes of IM injection or 5 minutes of IV administration.

- Prophylactic anticholinergic medications (but not amantadine) are reasonable when using high-potency FGAs (eg, haloperidol and fluphenazine), in young men, and in patients with a prior dystonia. Dystonia can be minimized by using lower initial doses of FGAs and by using SGAs instead of FGAs.

\section{b. Akathisia}

- Symptoms include subjective complaints (feelings of inner restlessness) and/or objective symptoms (pacing, shifting, shuffling, or tapping feet).

- Treatment with anticholinergics is disappointing. Reduction in antipsychotic dose may be the best intervention. Alternatively, switch to an SGA, although akathisia occasionally occurs with the SGAs. Quetiapine and clozapine appear to have the lowest risk. Benzodiazepines may be used, but not in patients with a history of substance abuse. Propranolol (up to $160 \mathrm{mg} /$ day), nadolol (up to 80 $\mathrm{mg}$ /day), and metoprolol (up to $100 \mathrm{mg} /$ day) are reported to be effective.

\section{c. Pseudo parkinsonism}

- Patients with pseudo Parkinsonism may have any of four cardinal symptoms:

$\checkmark$ Akinesia, bradykinesia, or decreased motor activity, including mask-like facial expression, micrographia, slowed speech, and decreased arm swing

$\checkmark$ Tremor-predominantly at rest; decreasing with movement

$\checkmark$ Rigidity-stiffness; cogwheel rigidity is seen as the patient's limbs yield in jerky, ratchet-like fashion when moved passively by the examiner.

$\checkmark$ Postural abnormalities-stooped, unstable posture and slow, shuffling, or festinating Gait.

- Risk factors-FGAs (especially in high dose), increasing age, and possibly female gender.

- Accessory symptoms-seborrhea, sialorrhea, hyperhidrosis, fatigue, weakness, dysphagia, and dysarthria.
- The onset of symptoms is usually 1 to 2 weeks after initiation of antipsychotic therapy or dose increase. The risk of pseudo parkinsonism with SGAs is low except with risperidone in doses greater than $6 \mathrm{mg} /$ day.

- Benztropine has a half-life that allows once- to twice-daily dosing. Typical dosing is 1 to $2 \mathrm{mg}$ twice daily up to a maximum of $8 \mathrm{mg}$ daily. Trihexyphenidyl, diphenhydramine, and biperiden usually require three-times-daily dosing. Diphenhydramine produces more sedation. All of the anticholinergics have been abused for euphoriant effects.

- Amantadine is as efficacious as anticholinergics with less effect on memory.

- Attempt to taper and discontinue these agents 6 weeks to 3 months after symptoms resolve.

\section{d. Tardive Dyskinesia}

- Tardive Dyskinesia (TD) is characterized by abnormal involuntary movements occurring with chronic antipsychotic therapy.

- The classic presentation is buccolingual-masticatory (BLM) or orofacial movements. Symptoms may become severe enough to interfere with chewing, wearing dentures, speech, respiration, or swallowing. Facial movements include frequent blinking, brow arching, grimacing, upward deviation of the eyes, and lip smacking. Restless choreiform and athetotic movements of the limbs occur in later stages. Movements may worsen with stress, decrease with sedation, and disappear with sleep.

- Screen at baseline and at least quarterly using the Abnormal Involuntary Movement Scale (AIMS) and the Dyskinesia Identification System:

\section{Condensed User Scale (DISCUS) to detect TD.}

- Dosage reduction or discontinuation may reduce symptoms, and some patients may have complete disappearance of symptoms if implemented early in the course of TD.

- Prevention of TD- (1) use SGAs as first-line agents;

2 Use the DISCUS or other scales to assess for early signs of TD at least quarterly; and

3 Discontinue antipsychotics or switch to SGAs at the earliest symptoms of TD, if possible.

- Risk factors for TD-duration of antipsychotic therapy, higher dose, possibly cumulative dose, increasing age, occurrence of acute extrapyramidal symptoms, poor antipsychotic response, and diagnosis of organic mental disorder, diabetes mellitus, mood disorders, and possibly female gender.

- Switching to clozapine is a first-line strategy in patients with moderate to severe dyskinesias $24,25,26,27$.

\section{B. Anti-cholinergic effects}

Anti-cholinergic effects include constipation, urinary retention, dry mouth, blurred vision. These effects are common with low potency first generation antipsychotics and clozapine. Anticholinergic side effects, most likely to occur with low potency FGA, clozapine, and olanzapine, include impaired memory, dry mouth, constipation, tachycardia, blurred vision, inhibition of ejaculation, and urinary retention. Elderly patients are especially sensitive to these side effects. Dry mouth can be managed with increased intake of fluids, oral lubricants (Xerolube), ice chips, or the use of sugarless chewing gum or hard candy. 
Constipation can be treated with increases in exercise, fluid, and dietary fiber intake. $28,29,30$

\section{Hyperprolactinemia}

It occurs mostly within a few weeks of beginning of treatment or increasing the dosage but can also arise after long-term stable use. It is a common with the use of any first-generation antipsychotic drugs as well as second generation antipsychotic drugs such as Risperidone and is dose dependent. An increase in prolactin levels may cause by blocking of dopamine in hypothalamus and it results, galactorrhoea in men. Hence, physicians routinely measure the serum prolactin levels. ${ }^{31,32}$

\section{Cardiovascular}

All antipsychotic may cause ECG changes such as prolonged QT interval and orthostatic hypotension. Orthostatic hypotension occur with the low-potency first generation antipsychotic drugs such as Chlorpromazine, thioridazine and with second generation antipsychotic drugs such as Clozapine, Resperidone, Olanzepine, Quetiapine. Antipsychotics most likely to cause ECG changes are low potency first generation antipsychotic drug such as thioridazine and the second-generation antipsychotic drug such as Ziprasidone. $33,34,35,36$

\section{E. Sexual Dysfunction}

Sexual dysfunction refers to as reduction in desire or libido, diminished arousal, decline in the frequency of intercourse. It is very common and up to $49 \%$ of patients taking antipsychotic drugs report problems with sexual dysfunction, a distressing adverse effect that can lead to poor medication adherence. Both FGAs and SGAs drugs can impair arousal and orgasm in men and women. Galactorrhea in women and men also gynecomastia in men are more common with second generation antipsychotics and with resperidone and can be dos e related. ${ }^{37,38,39}$

\section{F. Weight Gain}

The common adverse effect of antipsychotic drugs are weight gain which can be rapidly increase and difficult to control. It doesn't dose-dependent and substantially significant side effect of antipsychotic drugs and mainly reported in adults and children. Olanzepine and Clozapine may cause more weight gain as compared to other atypical antipsychotic drugs such as $>7 \%$ of the baseline bodyweight in $40 \%$ or more of patients. It can also induce cardiovascular and cerebrovascular morbidity and mortality reduced quality of life and poor drug compliance. The first-generation antipsychotic drugs such as Chlorpromazine and thioridazine cause high risk of weight gain but risk is greatest with use of second-generation antipsychotic drugs such as Clozapine and Olanzepine. Antipsychotic drugs such as Quetiapine, Resperidone, Paliperidone, Sertindole, Zotepine have intermediate risk of weight gain. These are the factors which are responsible for the risk of weight gain due to antipsychotic drugs at redemographic variables, treatment settings, illness characteristics, history and current treatment with antipsychotics and other drugs etc. $40,41,42$

\section{G. Neuroleptic Malignant Syndrome}

A life-threatening reaction may cause by the use of antipsychotic drugs but it is rare. It can be initiated within first week of treatment of antipsychotics but can develop at any time. NMS is characterized by labile blood pressure, tachypnoea, tachycardia, sialorrhea, and flushing, skin pallor. The idiosyncratic reactions to antipsychotic drugs include cardiopulmonary arrest, aspiration pneumonias, myoglobinuric renal failure with special reference to Clozapine. Up to $9 \%$ of total death rate was reported and out of 177 cases $20 \%$ of cases of NMS with patients aged 18 years or younger were reported as serious adverse drug reactions. $43,44,45$

\section{H. Sedation And Cognition}

- Administration of most or the entire daily dose at bedtime can decrease daytime sedation and may eliminate the need for hypnotics.

- Compared to FGAs, the SGAs have shown cognitive benefits. However, the CATIE trial showed no difference in cognitive improvement between SGAs and the FGA perphenazine ${ }^{1,2}$.

\section{Seizures}

- All patients treated with antipsychotics have an increased risk of seizures. The highest risk for antipsychotic-induced seizures is with the use of chlorpromazine or clozapine. Seizures are more likely with initiation of treatment and with higher doses and rapid dose increases.

- When an isolated seizure occurs, a dosage decrease is recommended, and anticonvulsant therapy is usually not recommended.

- If a change in antipsychotic therapy is required, risperidone, thioridazine, haloperidol, pimozide, trifluoperazine, and fluphenazine may be considered. ${ }^{1}$

\begin{tabular}{|c|c|c|c|c|c|c|}
\hline \multicolumn{7}{|c|}{ Nedscapeo mmw.medscape.com } \\
\hline Drug & $\begin{array}{l}\text { Daily } \\
\text { dose (mg) }\end{array}$ & EPS & TD & $\begin{array}{l}\text { Hyper- } \\
\text { Prolactinemia }\end{array}$ & $\begin{array}{l}\text { Weight } \\
\text { gain }\end{array}$ & $\begin{array}{l}\text { Giucose } \\
\text { intolerance }\end{array}$ \\
\hline \multicolumn{7}{|c|}{ First-generation antipsychotics } \\
\hline Chlorpromazine & $50-450$ & ++ & ++ & + & ++ & ++ \\
\hline Haloperidol & $3-6$ & +++ & ++ & ++ & + & + \\
\hline Perphenazine & $6-48$ & $++1+++$ & ++ & + & + & ++ \\
\hline \multicolumn{7}{|c|}{ Second-generation antipsychotics } \\
\hline Clozapine & $150-600$ & \pm & \pm & \pm & ++ & +++ \\
\hline Risperidone & $2-6$ & $\pm \sim+$ & + & ++ & + & + \\
\hline Olanzapine & $10-20$ & $\pm \sim+$ & + & \pm & +++ & +++ \\
\hline Quetiapine & $150-750$ & \pm & + & \pm & + & ++ \\
\hline Ziprasidone & $80-200$ & $\pm \sim+$ & + & \pm & \pm & \pm \\
\hline Amisulpride & $50-800$ & $\pm \sim+$ & + & ++ & + & + \\
\hline Aripiprazole & $6-30$ & $\pm \sim+$ & + & \pm & \pm & \pm \\
\hline Perospirone & $12-48$ & $\pm \sim+$ & NA & \pm & \pm & \pm \\
\hline Blonanserin & $8-24$ & $\pm \sim+$ & NA & \pm & \pm & + \\
\hline
\end{tabular}




\section{J. Other Side effects of Antipsychotic Drugs}

Some of the ADRs are very common but discussion and reporting are very little such as constipation, fever, hypersalivation, nausea, colitis, delirium, eosinophilia, heat stroke, hepatic failure, pancreatitis, nocturnal enuresis. Clozapine is the most common agent which is responsible for the constipation. It can be severe and may lead to serious problems such as ileus, bowel occlusion and sometimes may be death. 1,2

\section{Implications and Management}

The burden of ADRs with the use of psychiatric drugs varies among studies. The range of implicated agents is diverse but antidepressant and antipsychotics drugs are most commonly associated with ADRs. These are the key points which is helpful in management of ADRs and facilitating the wellbeing of patients ${ }^{46,47,48}$ -

a. Clinicians have to able aware about all the ADRs which are associated from antipsychotic drugs.

b. The need to balance the potential risks with expected benefits has to be kept in mind by clinicians, Treatment is individualized as per patient basis such as diagnosis, age, physical status, other factors like co-morbid conditions, other history of medications, patient past response, nutritional status and so on.

c. Clinicians should be keep in mind that medication prescriptions should be accompanied by appropriate device.

d. When a patient on antipsychotic drugs, frequent assessment of metabolic parameters, ECG have to done.

e. Finally, keep in mind a current literature of patients will help a clinician better formulate a treatment plan, anticipate potential problem, and avoid them.

\section{ROLE OF THE CLINICAL PHARMACIST}

Since therapeutic compliance rates are so low and side effects are varied, clinical pharmacists have an important role to play in the management of schizophrenia. A recent study showed that $74 \%$ of schizophrenic patients discontinued their medication (medical adherence) within 1.5 years. ${ }^{52}$ The likelihood that a patient will develop a particular side effect depends upon the patient's vulnerability as well as the dose being administered and other co-administered medications. ${ }^{51}$ Clinical Pharmacists can monitor side effects and hence improve compliance by inquiring about positive and negative symptoms when the patient refills prescriptions, and communicate regularly with the patient's other health care professionals about changes in symptoms. In addition to ensuring the correct dosing of appropriate drugs, clinical pharmacists are key in guiding patients about where they can obtain further help (e.g., through individual or group therapy).

While existing antipsychotic medications are often effective for treating positive symptoms, they have little impact on negative symptoms and cognitive deficits. Therefore, there is a need to develop drugs that will allow for patient-specific treatment of symptoms. For example, lurasidone is a newer SGA that may be effective for cognitive symptoms. Additionally, while the newer therapies have addressed the issue of motor side effects, safety, and tolerability, there are increased concerns regarding weight gain and metabolic abnormalities. 53

\section{General Procedures for Manage ADRs-}

i) Lower the dose - When a antipsychotic drug provided benefit to the patients the ADR is also occur but it is dosedependent. So, the lowest dose is prescribed to the patients for achieving treatment goals.

ii) Switch to an antipsychotic drug - It can be relevant when the dosage adjustment cannot be proven beneficial for the patients and addressed a life- threatening or fatal.

iii) Prescribed a non- pharmacologic intervention - It proven effective when weight gain and related lipid abnormality was addressed so, the diet and exercise programs are effective.

iv) Treat with a concomitant medication - These are used for reducing the ADRs which is associated by antipsychotic drugs but they also have own adverse effects. Hence, few concomitant medication approaches are supported by evidence from randomized controlled trials ${ }^{49,50}$.

\section{CONCLUSION}

Occurrence of ADR had largely affected hospital stay of patients indirectly influencing economic burden on patients. ADR are often poorly identified and reported in day to day medical practice. As we collect more and more information about ADRs, we need an active surveillance system regarding identification and reporting of ADRs with antipsychotic drugs. Psychiatrist and clinical pharmacist are need to be made aware of these potentially fatal adverse effects associated with antipsychotic drugs via conduction of patients counseling (drugs, disease, doses \& side effects), quality-based seminars, published medical literature, conferences, learning programs and health care camps.

\section{REFERANCES}

1. Srinivasan R, Ramya G. Adverse Drug Reaction-causality assessment. Int J Res Pharm Chem. 2011; 1(3): 606-12.

2. Radua J,Cravaro VR, Ioannidis J.P.A. What causes psychosis? An umbreela review of risk and protective factors.Workld Psychiatry. 2018; 17: 49-66.

3. Haddad PM, Sharma SG. Adverse effects of atypical antipsychotics. CNS Drugs. 2007 Nov 1; 21(11): 911-36.

4. Chew ML, Mulsant BH, Pollock BG, Lehman ME, Greenspan A, Kirshner MA, Bies RR, Kapur S, Gharabawi G. A model of anticholinergic activity of atypical antipsychotic medications. Schizophrenia research. 2006 Dec 1; 88(1): 63-72.

5. Wallace M. Real progress-the patient's perspective. International clinical psychopharmacology. 2001 Jan 1; 16: S21-4.

6. Ghadirian AM, Chouinard G, Annable L. Sexual dysfunction and plasma prolactin levels in neuroleptic-treated schizophrenic outpatients. Journal of Nervous and Mental Disease. 1982 Aug.

7. Hamer S, Haddad PM. Adverse effects of antipsychotics as outcome measures. The British Journal of Psychiatry. 2007 Aug 1; 191(50): s64-70.

8. Meyer JM. Effects of atypical antipsychotics on weight and serum lipid levels. The Journal of clinical psychiatry. 2001; 62: 27-34

9. Haupt DW, Newcomer JW. Hyperglycemia and antipsychotic medications. Journal of Clinical Psychiatry. 2001 Jan 14; 62(27): 15-26.

10. Sussman N. Review of atypical antipsychotics and weight gain. The Journal of clinical psychiatry. 2001.

11. Iuppa CA, Nelson LA, Elliott E, Sommi RW. Adverse drug reactions: a retrospective review of hospitalized patients at a state psychiatric hospital. Hospital pharmacy. 2013 Nov; 48(11): 931-5.

12. Baldessarine, RJ. et al., 2006. Pharmacotherapy of psychosis and Mania. In Goodman and Gillman's: The Pharmacological basis of therapeutics (eds).Brunton LL, Lazo JS, Parker KL, New York, Mc GrawHill. Chapter 18: pp477-478. 
13. Howes OD, Murray RM. Schizophrenia: an integrated sociodevelopmental-cognitive model. The Lancet. 2014 May 10; 383(9929): 1677-87.

14. Divac N, Prostran M, Jakovcevski I, Cerovac N. Secondgeneration antipsychotics and extrapyramidal adverse effects. BioMed research international. 2014; 2014

15. Kuroki T, Nagao N, Nakahara T. Neuropharmacology of second-generation antipsychotic drugs: a validity of the serotonin-dopamine hypothesis. Progress in brain research. 2008 Jan 1; 172: 199-212.

16. Agid O, Mamo D, Ginovart N, Vitcu I, Wilson AA, Zipursky RB, Kapur S. Striatal Vs Extrastriatal Dopamine D 2 Receptors in Antipsychotic Response-A Double-Blind PET Study in Schizophrenia. Neuropsychopharmacology. 2007 Jun; 32(6): 1209.

17. Hedges D, Jeppson K, Whitehead P. Antipsychotic medication and seizures: a review. Drugs Today (Barc). 2003 Jul 1; 39(7): 551-7.

18. Potter WZ, Hollister LE. In: Katzung BG. Basic and Clinical Pharmacology. 9th ed. New York, NY: The McGraw-Hill; 2004: 462-481.

19. Casey DE. Implications of the CATIE trial on treatment: extrapyramidal symptoms. CNS spectrums. 2006 Jan; 11(S7): 25-31.

20. Ljungdahl A, Hanrieder J, Fälth M, Bergquist J, Andersson M. Imaging mass spectrometry reveals elevated nigral levels of dynorphin neuropeptides in L-DOPAinduced dyskinesia in rat model of Parkinson's disease. PloS one. 2011 Sep 30; 6(9): e25653.

21. Thornton E, Tran TT, Vink R. A substance P mediated pathway contributes to 6hydroxydopamine induced cell death. Neuroscience letters. 2010 Aug 30; 481(1): 64-7.

22. Casey DE. Pathophysiology of antipsychotic drug-induced movement disorders. The Journal of clinical psychiatry. 2004; 65: 25-8.

23. Shirzadi AA, Ghaemi SN. Side effects of atypical antipsychotics: extrapyramidal symptoms and the metabolic syndrome. Harvard review of psychiatry. 2006 Jan 1; 14(3): 152-64.

24. Tarsy D. Neuroleptic-induced extrapyramidal reactions: classification, description, and diagnosis. Clin Neuropharmacol 1983;6(Suppl. 1):S9-26.

25. Aguilar EJ, Keshavan MS, Martinez-Quiles MD et al. Predictors of acute dystonia in first-episode psychotic patients. Am J Psychiatry 1994; 151:1819-21.

26. M. Poznic Jesic, A. Jesic, J. Babovic Filipovic et al., -Extrapyramidal syndromes caused by antipsychotics, ||Medicinski Pregled, 2012; 65: 521-26.

27. Cohen BM, Keck PE, Satlin A, Cole JO. Prevalence and severity of akathisia in patients on clozapine. Biological Psychiatry. 1991 Jun 15; 29(12): 1215-9.

28. Van Harten PN, Hoek HW, Kahn RS. Acute dystonia induced by drug treatment. BMJ 1999; 319:623-6.

29. Seemuller F, Lewitzka $U$, Bauer $M$. The relationship of akathisia with treatment emergent suicidality among patients with first-episode schizophrenia treated with haloperidol or risperidone. Pharmacopsychiatry 2012; 45:292-6.

30. Ballenger JC. Evaluating the cost-effectiveness of reduced tardive dyskinesia with second-generation antipsychotics. Year Book of Psychiatry \& Applied Mental Health. 2009 Jan 1; 2009: 264-5.

31. Poyurovsky M. Acute antipsychotic-induced akathisia revisited. Br J Psychiatry 2010; 196:89-91.

32. Poyurovsky M, Pashinian A, Weizman R et al. Low-dose mirtazapine: a new option in the treatment of antipsychoticinduced akathisia. A randomized, double-blind, placebo- and propranolol-controlled trial. Biol Psychiatry 2006; 59:1071-7.

33. Kulkarni SK, Naidu PS. Pathophysiology and drug therapy of tardive dyskinesia: current concepts and future perspectives. Drugs Today (Barc). 2003 Jan 1; 39(1): 19-49.
34. Crismon ML, Argo TR, Buckley PF. Schizophrenia. In DiPiro JT, Talbert RL, Yee GC, et al, eds. Pharmacotherapy: A Pathophysiologic Approach. 7th ed. New York, NY: McGrawHill Inc; 2008: 1099-1122.

35. Chouinard G. Effects of risperidone in tardive dyskinesia: an analysis of the Canadian multicenter risperidone study. J Clin Psychopharmacol 1995;15:36S-44S.

36. Pouclet-Courtemanche H, Rouaud T, Thobois S et al. Longterm efficacy and tolerability of bilateral pallidal stimulation to treat tardive dyskinesia. Neurology 2016;86:651-9.

37. Saleh C, Gonzalez V, Coubes P. Role of deep brain stimulation in the treatment of secondary dystonia-dyskinesia syndromes. Handb Clin Neurol 2013;116:189-208.

38. Sobstyl M, Zabek M. Deep brain stimulation for intractable tardive dystonia: literature overview. Neurol Neurochir Pol 2016;50:114-22.

39. Lieberman, J.A., Tollefson, G., Tohen, M., Green, A.I., Gur, R.E., Kahn, R., McEvoy, J., Perkins, D., Sharma, T., Zipursky, R. and Wei, H., Comparative efficacy and safety of atypical and conventional antipsychotic drugs in first-episode psychosis: a randomized, double-blind trial of olanzapine versus haloperidol. American Journal of Psychiatry, 2003; 160(8): 1396-1404.

40. Thanvi B, Treadwell S. Drug induced parkinsonism: a common cause of parkinsonism in older people. Postgrad Med J 2009 Jun 1;85(1004):322-6.

41. Caligiuri MP, Lohr JB. Instrumental motor predictors of neuroleptic-induced parkinsonism in newly medicated schizophrenia patients. J Neuropsychiatry Clin Neurosci 1997;9:562-7.

42. Hriso E, Kuhn T, Masdeu JC et al. Extrapyramidal symptoms due to dopamine-blocking agents in patients with AIDS encephalopathy. Am J Psychiatry 1991; 148:1558-61.

43. Magnus RV. A comparison of biperiden hydrochloride (Akineton) and benzhexol (Artane) in the treatment of druginduced Parkinsonism. J Int Med Res 1980; 8:343-6.

44. Every-Palmer S, Ellis PM. Clozapine-induced gastrointestinal hypomotility: a 22-year bi-national pharmacovigilance study of serious or fatal 'slow gut' reactions, and comparison with international drug safety advice. CNS Drugs 2017; 31:699-709

45. Chen CY, Lane HY, Lin CH. Effects of antipsychotics on bone mineral density in patients with schizophrenia: gender differences. Clin Psychopharmacol Neurosci 2016; 14:238-49.

46. Madaan V, Dvir Y, Wilson DR. Child and adolescent schizophrenia: pharmacological approaches. Expert opinion on pharmacotherapy., 2008 Aug 1; 9(12): 2053-68.

47. Masi G, Liboni F. Management of schizophrenia in children and adolescents. Drugs., 2011 Jan 1; 71(2): 179-208.

48. Kwack YS, Ryu JS. Neuroleptic Malignant Syndrome in Children and Adolescents: A Review. Journal of the Korean Academy of Child and Adolescent Psychiatry, 2013; 24(1): 1320.

49. De Hert M, Hudyana H, Dockx L, Bernagie C, Sweers K, Tack J, Leucht S, Peuskens J: Second-generation antipsychotics and constipation: A Review of the Literature. European Psychiatry, 2011; 26: 34-44.

50. Taylor D, Paton C, Kapur S. The Maudsley prescribing guidelines in psychiatry. John Wiley \& Sons; 2015 Feb 23.

51. Tandon R. Antipsychotics in the treatment of schizophrenia: an overview. J Clin Psychiatry. 2011; 72(suppl 1):4-8.

52. Lieberman JA, Stroup TS, McEvoy JP, et al, for the Clinical Antipsychotic Trials of Intervention Effectiveness (CATIE) Investigators. Effectiveness of antipsychotic drugs in patients with chronic schizophrenia. N Engl J Med. 2005; 353:12091223.

53. Miyamoto S, Miyake N, Jarskog LF. Pharmacological treatment of schizophrenia: a critical review of the pharmacology and clinical effects of current and future therapeutic agents. Mol Psychiatry. 2012 May 15 [Epub ahead of print]. 